\title{
VARIA
}

\section{LES SÉRIES TÉLÉVISÉES À DESTINATION DES PUBLICS PRÉSCOLAIRES : UN MÉTA- GENRE TÉLÉVISUEL SPÉCIFIQUE}

\author{
Emilie Mondoloni ${ }^{1}$
}

Les séries télévisées à destination de la jeunesse et à fortiori à destination des publics préscolaires se sont récemment multipliées selon des formes et des contenus spécifiques. Le public des enfants âgés de trois à six ans est visé par une programmation ciblée ou non par les chaînes de télévision généralistes (TF1, France 5 et M6) et thématiques (Piwi, Playhouse Disney, Tiji et Cap canal) qui assurent la diffusion de ces séries télévisées spécifiques.

Une étude fondée d'une part sur l'analyse des grilles de programmation de deux chaînes généralistes (TF1 et France 5) et des chaînes thématiques citées précédemment, et d'autre part sur l'analyse d'un corpus de trois séries télévisuelles (Dora l'exploratrice, Oui-oui au pays des jouets et Jasper le pingouin), per-

1 Emilie Mondoloni est chercheur non-permanent au Gresec (Groupe de recherche sur les enjeux de la communication), Université Stendhal Grenoble 3.

Recherches en communication, $\mathrm{n}^{\circ} 34$ (2010). 
met de mettre en évidence un méta-genre audiovisuel spécifique au préscolaire.

Dans un premier temps, l'analyse des grilles de programmation permet d'établir des caractéristiques récurrentes des séries télévisées préscolaires : formats et procédés de production.

Dans un deuxième temps, l'analyse des séries télévisées montre la présence de plusieurs invariants dans celles-ci.

Aussi à partir de ces deux analyses, nous proposons une définition sémio-pragmatique du méta-genre préscolaire, désignant en cela les spécificités de celui-ci. Cette définition se décline à partir d'invariants observés dans les séries télévisées (relation temporelle du programme au monde, mode discursif et représentations des relations humaines et sociales), de critères de forme (formats et procédés de production) et un critère issu de la filière audiovisuelle (objectifs du contenu).

Les séries télévisées préscolaires intégrées dans la programmation télévisuelle des années 1960 (Bonne nuit les petits, par exemple) se sont récemment multipliées sur les chaînes généralistes (TF1, France 5 et M6) et thématiques (Piwi, Playhouse Disney, Tiji et Cap canal). Originales ou adaptées de produits culturels (livres, jeux, cartes postales etc.), ces séries télévisées se déclinent selon des formes et des contenus spécifiques.

Nous postulons que les séries télévisées à destination des publics préscolaires constituent des produits culturels spécifiques. Elles sont identifiables au sein d'un méta-genre ${ }^{1}$ audiovisuel spécifique que nous proposons ici de caractériser.

Plusieurs critères ont retenu notre attention dans l'identification du méta-genre préscolaire et nécessitent deux examens complémentaires : d'abord, l'analyse des grilles de programmation permet d'établir des caractéristiques récurrentes des séries télévisées préscolaires (formats et procédés de production) ; ensuite, l'étude de trois séries

1 Nous n'identifions pas ici un genre unique, mais plusieurs genres, au sein d'un méta-genre. Le méta-genre proposé à ce stade est ainsi une première proposition de taxinomie des séries télévisées préscolaires. 
télévisées (Oui-oui au pays des jouets ${ }^{1}$, Dora l'exploratrice ${ }^{2}$ et Jasper le pingouin ${ }^{3}$ ) montre la présence de plusieurs invariants dans cellesci. Par conséquent notre caractérisation d'un méta-genre préscolaire se situe à un double niveau : pragmatique et sémiologique.

Aussi, nous reviendrons dans un premier temps sur l'acception du genre et nous situerons par rapport à celle-ci. Dans un deuxième temps, nous développerons les résultats de notre étude de séries télévisées. Notre hypothèse de travail repose sur l'idée d'un temps télévisuel (Jost, 2007) spécifique dans les séries télévisées préscolaires, également sur une figure du lecteur (Eco, 1985) et le mode discursif du programme qui en découle, et sur le recours à des techniques de production audiovisuelle spécifiques (dimension pragmatique). A l'issue de ces observations, nous proposerons une typologie des genres préscolaires, dans un dernier temps.

\section{La polysémie du « genre »}

Le genre est une notion polysémique dont les sens diffèrent selon le contexte d'usage et les acteurs y faisant référence. Les chercheurs (en lettres, sciences de l'information et de la communication, cinéma notamment) proposent deux approches : les premières (Casetti, 1988 ; Jost, 1997 ; Soulages, 2007) désignant les stratégies des acteurs des industries culturelles, les autres désignant, à l'aide d'analyses sémiologiques, des taxinomies des produits audiovisuels (Bourdon, 1988 ; Charaudeau, 1997).

1 Créé en 1949, par la romancière Enid Blyton, Oui-oui, de son vrai nom Noddy (qui secoue la tête), est l'une des figures emblématiques des séries télévisées à destination des tout-petits. Actuellement produite par Chorion (http://www.chorion. co.uk/, consulté le 12 août 2009), la série est diffusée sur France 5 et Tiji.

2 Produit par Nickelodeon, la série américaine met en scène une jeune américaine d'origine hispanique, Dora. Dora se présente comme un programme ludo-éducatif. Toutefois, au même titre qu'Hélène et les garçons, il s'agit d'une série extrêmement critiquée dans la famille. Dora l'exploratrice est diffusé sur TF1 et Tiji.

3 Jasper est un dessin animé produit par Millimages, producteur français. Il a été diffusé sur France 5, WDR, ABC, Disney UK, Radio Canada, TV Ontario, Disney France, RTE (Irlande), NRK (Norvège), SVT (Suède), TV2 (Danemark), TVNZ (Nouvelle Zélande) et Playhouse Disney (http://www.millimages.com/site/tv/prod. php?lang=fr\&tv_id=11, consulté le 12 août 2009). 


\section{Le genre : composante des stratégies des industries culturelles}

La notion de genre coïncide avec les stratégies économiques et politiques de multiples acteurs. Pour certains auteurs, le genre employé dans cette dimension est un " pacte » ou un « contrat » entre l'émetteur et le récepteur, et sujet à de constants réajustements (Casetti, 1988). Par conséquent le genre télévisuel est ainsi écartelé entre le cadre d'usage des publics et « la mise en formule » (Soulages, 2007, p. 55) des producteurs et des programmateurs.

Patrick Charaudeau ajoute que les genres s'inscrivent « dans une relation sociale de reconnaissance, témoignant d'une codification qui leur est propre (propre à leur contexte socioculturel) et peut donc varier d'un contexte à l'autre (un talk show à la française se différencie d'un talk show à l'italienne) » (Charaudeau, 1997, p. 86). De plus, ces classifications induites par des stratégies institutionnelles de production et des pratiques professionnelles visent la mise en place sociale et la légitimité des produits audiovisuels ( $\mathrm{Nel}, 1997$, p. 36). Issues de l'activité de réception des téléspectateurs, elles garantissent à l'identité générique une marge de labilité (Nel, 1997, p. 36), un sceau attestant la composition du produit (Jost, 1997, p. 15).

Egalement le genre se développe au sein de trois lieux de « pertinence » (Charaudeau, 1997, p. 86) :

- Le lieu des conditions de production, c'est-à-dire celui dans lequel se trouve l' « instance d'énonciation » (la chaîne de télévision, la maison de production etc.),

- Le lieu des conditions d'interprétation, dans lequel se trouve «l'instance de réception » (le domicile du téléspectateur, une salle de cinéma etc.),

- Et le lieu de construction du discours, dans lequel se trouve « le texte comme produit fini » (Charaudeau, 1997, p. 86).

C'est à partir de ces trois lieux de pertinence qu'on peut analyser le genre dans sa dimension stratégique.

Ainsi la généricité est extrêmement complexe pour deux raisons majeures. D'une part, le genre pousse à respecter les contraintes de la culture de flot : accord entre temps télévisuel et temps réel, accord entre les stratégies d'industrialisation et la demande de plus en plus forte des téléspectateurs, étude des scores d'audience et de la concurrence (Nel, 1997, p. 42). D'autre part, la généricité exige la présence constante d'invariants afin d'assurer l'identification des genres, alors 
que l'impératif de création implique un renouvellement des formes ou du moins « d'explorer des stratégies de variation » (Nel, 1997, p. 44). En d'autres termes, les limites des genres sont repoussées par les impératifs de création.

Enfin les stratégies économiques et politiques des genres se caractérise par une « promesse qui entraîne chez le spectateur des attentes, que la vision du programme met à l'épreuve » (Jost, 1997, p. 15). L'acte de nommer et de classer un programme selon un genre relève donc de stratégies (Jacquinot \& Leblanc, 1996 ; Jost, 2009) de la part des diffuseurs. D'ailleurs les critères de classement sont hétérogènes, faisant parfois référence au contenu des émissions (policier par exemple), à leur mode de production (dessin animé) ou même au public visé (émissions destinées à la jeunesse) (Jacquinot \& Leblanc, 1996, p. 46).

\section{Le genre : composante sémiologique}

La tradition littéraire propose depuis 1'Antiquité des catégories définies à l'aide de critères variables : des critères de formes, de périodes historiques, des critères relatifs à la structure du texte et à son dispositif énonciatif (Charaudeau, 1997, p. 81).

Depuis les années 1920 en France, les débats n'ont cessé d'être animés sur la notion de genre et de ce qu'elle revêt de sens au sein des courants littéraires. Et d'abord la théorie littéraire rattache le genre et sa détermination à la fois au cas singulier et à une reconnaissance au sein d'une généralité. Dominique Combe relève ainsi :

Le « sceau » ou la « signature » qui inscrit l'œuvre dans le singulier par la « manière » de l'artiste est en effet indissociable des genres, de leurs conventions, de leurs contraintes et de leur hiérarchie (Combe, 2002, p. 34).

Les genres comportent également des invariants tels les modes discursifs. Cependant, les genres ne sont pas des classes statiques d'œuvres, mais des lignées dynamiques qui transforment la littérature, véritables moteurs de 1'《évolution littéraire » (Combe, 2002, p. 46).

Aussi les auteurs de la théorie littéraire ont temporairement conclu qu'il n'existe aucuns traits définitoires stables des genres qui instaureraient un code générique univoque. On s'accorde donc à reconnaître que les genres constituent des repères afin de reconnaître et de distinguer des œuvres. 
Bien que ces repères soient nécessaires à l'intelligibilité des objets du monde (Charaudeau, 1997, p. 82), Catherine Kerbrat-Orecchioni souligne l'artefact du genre :

Ce terme de genre dénote un artefact, un objet construit par l'abstraction généralisante, à partir des objets empiriques que sont les textes, qui ne sont jamais que des représentants impurs de tel ou tel genre (Kerbrat-Orrecchioni, 1980, in Nel, 1997, p. 35).

Ces deux points de vue complémentaires engagent à s'interroger sur les deux dimensions du genre : une dimension épistémologique et une dimension stratégique.

La dimension épistémologique définit les genres télévisuels selon plusieurs critères. Jérôme Bourdon propose une sémiologie des genres audiovisuels d'après trois partages génériques (Bourdon, 1988, p. 21) : fiction/non-fiction, continuité/montage, et l'étude des genres historiques attestés.

Or ces propositions ne s'appliquent pas à notre cas, car une majorité pour ne pas dire la quasi-totalité des programmes destinés aux publics préscolaires sont des fictions. Ensuite le deuxième partage (continuité/ partage) perd également sa pertinence, puisque le principe même des séries destinées aux tout-petits repose sur le montage. Enfin le troisième partage générique nous paraît insuffisant car il repose aujourd'hui sur des stratégies économiques et politiques. Il doit être envisagé avec précautions car il n'est jamais (ou rarement) fondé sur une caractéristique objective du produit audiovisuel. Aussi indiquerons-nous notre propre grille de lecture des programmes préscolaires dans le but d'en proposer une taxinomie.

\section{Un méta-genre audiovisuel spécifique}

L'analyse des séries télévisées préscolaires a porté sur trois séries $^{1}$ : Dora l'exploratrice, Oui-oui au pays des jouets et Jasper le

1 L'analyse des séries télévisées a été menée à l'Inathèque de Paris. La série Oui-oui au pays des jouets a fait l'objet d'un corpus plus conséquent : 40 épisodes de Ouioui diffusés de mars 2003 à juillet 2003 et 17 épisodes diffusés de décembre 2005 à janvier 2006 sur France 5 ; 40 épisodes de la première version du dessin animé, diffusés entre janvier 1995 et novembre 1995 sur France 3, complétés de 12 épisodes 
pingouin : soit 61 épisodes de Dora diffusés entre 2003 et 2006 sur TF1, 171 épisodes de Oui-oui correspondant à trois versions différentes de la série et diffusés entre janvier 1995 pour les premiers et mars 2006 pour les derniers, enfin 52 épisodes de Jasper le pingouin diffusés entre mars 2003 et octobre 2005 sur France 5.

\section{La relation temporelle du programme au monde}

Le temps télévisuel repose sur la production de croyances qui confèrent au temps des programmes un degré d'existence plus ou moins grand (Jost, 2007, p. 35) et génère un horizon d'attente, une disposition d'esprit du téléspectateur. François Jost propose ainsi d'identifier le temps télévisuel à partir de trois types de relation temporelle du programme au monde, en s'appuyant sur les travaux Charles S. Peirce ${ }^{1}$.

Le temps indiciel d'abord, regroupe tous les genres qui nous mettent directement en contact avec le temps de notre monde. Le temps iconique caractérise ces programmes qui construisent un temps, parfois proche du nôtre, mais dont l'existence n'est pas assurée. Enfin le temps symbolique confère une signification particulière à la temporalité du document quand il est regardé comme une œuvre (Jost, 2007, p. 38). Sur la base de cette catégorisation, nous pouvons maintenant explorer les rapports au temps développés par les fictions qui constituent notre corpus.

D'abord, aucune confusion n'est possible entre le monde de Oui-oui et le monde réel, même si le déroulement de la série se calque sur celui d'une journée. Par conséquent, la série se situe dans une certaine atemporalité. Pour les diffuseurs, chaque épisode est susceptible de faire l'objet d'une rediffusion sans que la place de celui-ci dans la grille de programmation ne soit importante pour la compréhension de l'histoire.

Oui-oui mobilise un temps iconique, qui requière une forte attention spectatorielle, mais aussi un temps symbolique, reposant sur sa structure propre. En effet, le temps réel d'une journée dure 24 heures, là où un épisode de la série dure 11 minutes. Toutefois, certains épisodes peuvent poser des problèmes de programmation pour les diffuseurs (ex : les épisodes situant leurs récits à Noël). Les épisodes visionnés sur

diffusés de 2001 à 2005 sur France 5 ; enfin 52 épisodes de la série J'apprends l'anglais avec Oui-oui, diffusés sur France 5 de décembre 2005 à mars 2006.

1 Charles S. Peirce, sémioticien, a travaillé sur les notions d'icône, d'indice et de symbole pour distinguer les relations des signes aux objets qu'ils représentent. 
le sujet sont systématiquement programmés un à deux jours avant Noël, voire le jour même. Ils s'inscrivent alors dans un temps indiciel, tout en se situant dans un lieu imaginaire.

Ces constats sont identiques pour la série Jasper le pingouin : les temps symbolique et iconique sont majoritairement employés. Trois épisodes de la série se situent toutefois dans un temps indiciel : «Le carnaval », programmé le 2 avril 2003, « Joyeuses Pâques », programmé le 28 mars 2005 et « Halloween », programmé le 31 octobre 2005. Enfin, l'étude de la série télévisuelle Dora l'exploratrice confirme nos observations des deux précédentes séries : les temps iconique et symbolique sont les plus employés.

Ainsi le temps indiciel est rarement employé dans les séries télévisées à destination des téléspectateurs préscolaires. Cela nous indique un rapport plus distancié des contenus à la réalité, que les programmes tout-public. Ceci constitue une caractéristique indiquant la spécificité des produits audiovisuels à destination des tout-petits : temps iconique et temps symbolique sont récurrents et dominants dans les trois séries.

A présent, nous nous proposons d'analyser le mode discursif des séries préscolaires à partir des mouvements des lecteurs dans l'écriture du texte et de la figure de « lecteur modèle » proposée par Umberto Eco (1985).

\section{Les mouvements des lecteurs dans l'écriture du texte}

La théorie du texte d'Umberto Eco, selon laquelle le texte présuppose chez son lecteur des connaissances sur la réalité et des compétences textuelles (toutefois restreintes chez le téléspectateur préscolaire), éclaire d'après nous l'identification du méta-genre préscolaire par les mouvements des jeunes téléspectateurs dans l'écriture du texte. Ainsi le texte dégage un « lecteur modèle » (Eco, 1985), figure caractérisée par une structure du texte spécifique qui « tel qu'il apparaît dans sa surface (ou manifestation) linguistique, représente une chaîne d'artifices expressifs qui doivent être actualisés par le destinataire » (Eco, 1985, p. 61). Egalement le texte est un système contrôlant sa propre interprétation et proposant des « jalons » au lecteur, qui vit sur la plusvalue de sens introduite par le destinataire (Eco, 1985, p. 63-64).

L'intérêt de ces travaux dans le cas des séries préscolaires réside dans la proposition de cadres d'interprétation interne au texte, cadres notamment observés dans Dora l'exploratrice. La « carte» ne constitue- 
t-elle pas un jalon pour le jeune téléspectateur le guidant dans sa lecture du programme?

Par ailleurs, les séries préscolaires assimilent les lecteurs/téléspectateurs dans la constitution des contenus. Oui-oui au pays des jouets et Dora l'exploratrice intègrent directement les téléspectateurs dans l'écriture, dans ce qu'Umberto Eco désigne comme « des mouvements coopératifs et conscients de la part du lecteur » (Eco, 1985, p. 62). Les personnages principaux des séries demandent aux enfants d'accomplir des gestes, de répéter des expressions (Dora l'exploratrice), ou sont parfois invités à la réflexion (Oui-oui notamment). En d'autres termes, les publics préscolaires coopèrent à l'écriture de leurs séries, aidant ainsi leurs séries à fonctionner (Eco, 1985).

Plus que n'importe quels programmes tout-public, les produits audiovisuels préscolaires requièrent une écriture spécifique, car ils reposent sur les capacités propres aux membres de l'audience auxquels ils se destinent (Eco, 1985, p. 64). L'écriture des séries télévisées présuppose chez le téléspectateur préscolaire une coopération de celuici à l'écriture de l'épisode, qui s'appuie sur un mode discursif recourant à l'interpellation des tout-petits.

Après avoir montré la relation temporelle des programmes préscolaires au monde, puis - dans la partie présente - le mode discursif spécifique, nous nous intéressons à présent à des critères pragmatiques d'observation du méta-genre préscolaire.

\section{Le méta-genre préscolaire : prédominance de l'animation et des formats courts}

Les résultats présentés à présent sont issus de 1'étude des grilles de programmation de TF1, France 5, Piwi, Tiji, Playhouse Disney et Cap canal, pour la semaine du 7 au 13 septembre 2009.

Une première comparaison entre les deux chaînes hertziennes montre que $43,75 \%$ des programmes jeunesse de TF1 et $70 \%$ des programmes de France 5 ont une durée inférieure à 15 minutes. Pour France 5, les téléspectateurs préscolaires constituent une cible spécifique à laquelle la chaîne adapte la construction de la programmation. TF1, pour sa part, cherche davantage à rassembler les audiences, ce qui explique le recours à des formats plus longs (qui intéresseront les plus grands). 
Le type de production majoritaire dans les grilles des deux chaînes est l'animation. Cette constante entre France 5 et TF1 explique pour partie la représentation des produits audiovisuels préscolaires, d'après ces mêmes publics. En effet, les grilles de programmation jeunesse " autorisées » par les parents ou la structure familiale comprennent majoritairement des dessins animés.

L'analyse des grilles de programmation des chaînes thématiques montre la prédominance de l'animation dans les grilles (à l'exception de Cap canal), entre 86,20\% des programmes de Piwi et 92,6\% de Playhouse Disney. Entre 79,31 \% (Piwi) et 88,88 \% (Tiji) des formats sont inférieurs ou égaux à 15 minutes. Aucune chaîne ne diffuse en continu, au contraire des autres chaînes thématiques.

Par ailleurs, une stratégie récurrente des programmateurs consiste en la mutidiffusion des programmes dans la même journée, rendant ces programmes plus rentables et par conséquent plus vite amortis pour ces chaînes.

\section{Typologie des genres préscolaires}

Les formats spécifiques des séries télévisées préscolaires ne recouvrent pas ceux des programmes tout-public et soulèvent la difficulté pour les chaînes généralistes d'intégrer des émissions jeunesse dans leurs grilles.

Centrées majoritairement sur l'animation, les fictions se différencient par les caractéristiques graphiques, la structure narrative, les personnages et les sujets abordés. A l'évidence, ces caractéristiques démontrent que l'on doit bien distinguer un méta-genre télévisuel spécifique.

Les programmes préscolaires reposent sur les caractéristiques d'un méta-genre identifiable suivant le tableau récapitulatif que nous proposons ici (Tableau 1). Trois familles de critères permettent d'identifier les genres des séries télévisées préscolaires : les critères observables par analyse des séries (comprenant la relation temporelle du programme au monde, le mode discursif ${ }^{1}$ et les représentations des relations humaines

1 En effet, le lecteur préscolaire est interpelé par le programme qui pose des « jalons » pour faciliter sa lecture et maintenir son attention. 


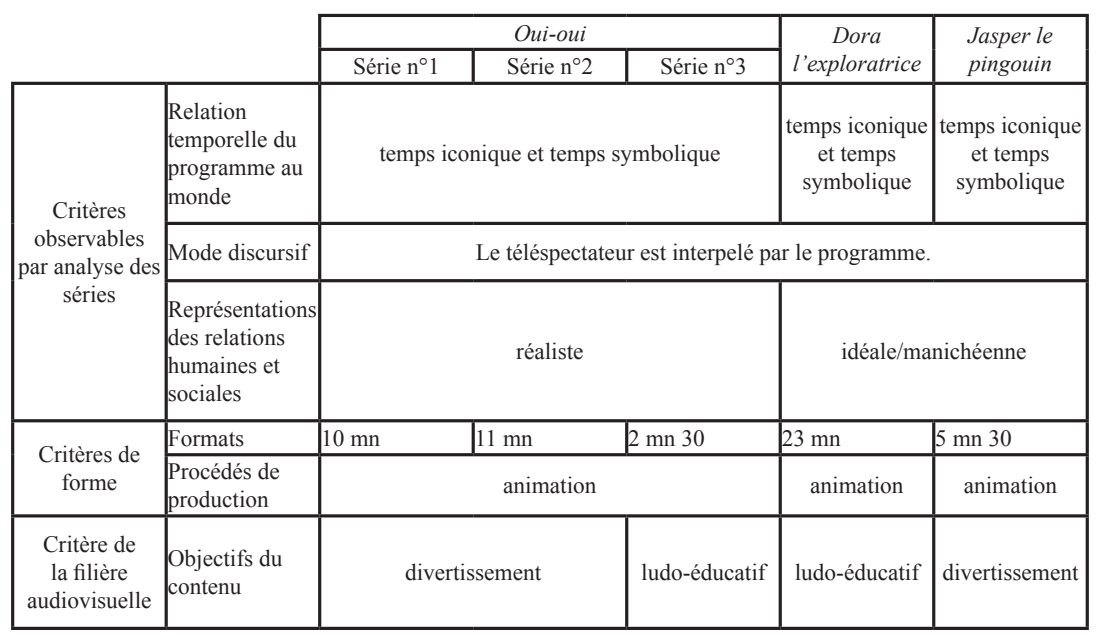

Tableau 1 : Typologie des genres préscolaires

et sociales), des critères de forme (formats et procédés de production) et un critère lié à la filière audiovisuelle (les objectifs des contenus présentés par les producteurs) ${ }^{1}$.

Le méta-genre des séries télévisées préscolaires se caractérise par une relation temporelle des programmes au monde représentée par les temps iconique et symbolique. On peut faire l'hypothèse que le recours à ces deux temps permettrait aux enfants d'identifier les séries et leurs contenus comme différents de notre monde.

L'analyse des trois séries télévisées montre un deuxième invariant au sein de ce méta-genre : le mode discursif. A partir de l'étude des mouvements du lecteur dans l'écriture et l'interprétation des séries télévisées, nous montrons que l'originalité des programmes préscolaires réside dans leur mode discursif, où l'on peut voir que ceux-ci interpellent le jeune téléspectateur. Une lecture rapide d'autres séries télévisées indique que ce critère se vérifie auprès d'autres séries (telles La maison de Mickey, Adibou aventure, 1, 2, 3 BO, 64 rue du Zoo ou Clifford le gros chien rouge) et constitue un invariant.

Le troisième critère de classement des produits audiovisuels divise le méta-genre en deux genres selon les représentations des relations humaines et sociales : un genre dit « réaliste » (représenté par Oui-oui

1 D'autres critères n'ont pas été retenus par manque de pertinence : par exemple la référence à des « genres » télévisuels et cinématographiques canoniques tel le genre policier. 
dans notre corpus) et un genre dit « idéal/manichéen » (Dora l'exploratrice et Jasper le pingouin).

Le méta-genre préscolaire est reconnaissable à la spécificité des formats des séries qui le composent. Les formats des séries télévisuelles ne font nullement référence à ceux des programmes tout-public. Pour rappel, entre 79,31\% (pour Piwi) et 88,88 \% (pour Tiji) des programmes des chaînes thématiques ont un format inférieur ou égal à quinze minutes. Ce critère peut constituer l'un de ceux à partir duquel la grille fluctue, du fait de l'existence de formats supérieurs à celui indiqué.

Les grilles de programmation indiquent que les programmes jeunesse à destination des tout-petits sont caractérisés par quatre types de produits ${ }^{1}$ : les dessins animés (majoritairement employés dans la création de séries télévisuelles préscolaires), les séries, les émissions et les magazines. Les dessins animés constituent $86,20 \%$ des programmes de Piwi, et 92,60\% des programmes de Playhouse Disney. Toutefois ce critère seul ne détermine pas l'appartenance du produit audiovisuel au méta-genre préscolaire. En d'autres termes, un dessin animé ne constitue pas nécessairement un produit du méta-genre préscolaire.

Enfin, nous avons intégré un dernier critère reposant sur les déclarations et stratégies des acteurs de la filière audiovisuelle : les objectifs des contenus. Présentés dans la presse professionnelle, les magazines télévisuels, les sites internet des chaînes, ceux des producteurs et des séries télévisées, ces objectifs sont communiqués auprès des professionnels et des parents comme l'élément d'un argumentaire de vente de la série télévisée. Ces différents acteurs classent ainsi les programmes selon deux objectifs (divertir, souvent identifié sous le terme « ludique», et éduquer), à partir desquels nous identifions trois genres : les divertissements, le genre éducatif et le genre dit « ludo-éducatif » par les producteurs (qui divertissent et éduquent).

Cette proposition de taxinomie présente un cadre rigide. Ce travail constitue en effet une première étude du sujet et nécessite d'être complété par l'étude approfondie de plusieurs critères (notamment la question des différences de réalisation et d'écriture en fonction du pays

1 Nous reposons ce classement sur l'étude des grilles de programmation des chaînes thématiques Piwi, Playhouse Disney et Tiji, pendant la semaine du 7 au 13 septembre 2009. 
d'origine. Ceci peut-il alors contribuer à dégager un invariant au sein du méta-genre ?). Par ailleurs, plusieurs critères sont flexibles, particulièrement celui des procédés de production (où nous n'avons pas intégré les séries de marionnettes et en costume, aujourd'hui peu présentes dans les grilles de programmation françaises comparativement aux grilles britanniques par exemple). On peut également ajouter que le tableau présenté ci-dessous regroupe les invariants étudiés à ce jour, sans proposer de dénomination stricte de genre et laissant cette taxinomie ouverte à d'autres évolutions.

\section{Conclusion}

Plusieurs limites peuvent être observées dans cette étude et constituent des perspectives de recherche. D'abord l'étude des séries télévisées a porté uniquement sur des séries réalisées en animation. Or il existe d'autres produits audiovisuels proposés aux enfants préscolaires. Leur étude permettrait de vérifier ou d'infirmer, et de compléter cette première approche : sur les formats des séries télévisées proposées aux tout-petits, sur les critères d'identification du méta-genre décrit ci-dessus. Par ailleurs, cet état des lieux n'est pas exhaustif et l'étude se limite au territoire français et aux chaînes conventionnées par le CSA. Or d'autres chaînes thématiques étrangères diffusent des séries télévisées à destination des plus jeunes (BabyFirst, BabyTV, Boomerang TV, Nickelodeon etc.). La question des formats et de la diversité des produits audiovisuels se pose également sur ces chaînes, bien que l'on ait pu observer la prédominance des séries d'animation.

De plus, cette étude ne traite en aucun cas la question des stéréotypes, qui sont présents dans tous les programmes télévisées (destinés aux enfants et à d'autres téléspectateurs). Les discours d'acteurs publics, d'éducateurs, de parents également décrivent les séries enfantines comme vecteurs de stéréotypes. Toutefois, l'étude des stéréotypes ne nous a pas semblé pouvoir être intégrée à l'étude des genres audiovisuels préscolaires, car l'objectif de l'étude était de montrer les spécificités des séries télévisées préscolaires et les stéréotypes étant présents dans tous types de programmes à destination de tous les publics, leur simple présence ne constitue pas une spécificité des programmes préscolaires. Par ailleurs les grilles de recueil et d'analyse du corpus se sont avérées insuffisantes pour traiter cette question. 
Enfin il s'agit d'un travail d'analyse des programmes qui pose des questions sur leur réception, auxquelles une première étude (Mondoloni, 2011) menée à ce jour répond partiellement. Par conséquent, une autre piste de recherche pourrait aller vers des études de réception.

Ces résultats proposent ainsi une première identification des séries télévisuelles au sein d'un méta-genre, dont les critères d'identification seront amenés à évoluer, non pas seulement du fait de l'évolution des séries elles-mêmes, mais aussi et surtout des travaux à venir.

\section{Références :}

Bourdon, J. (1988). Propositions pour une sémiologie des genres audiovisuels. Quaderni, 4(4), 19-36.

Casetti, F. (1990). Tra me e te. Rome : Rai-VPT. 1988, trad. fr. d'un chapitre. Réseaux, 44-45.

Charaudeau, P. (1997). Les conditions d'une typologie des genres télévisuels d'information. Réseaux, 81(15), 79-101.

Combe, D. (2002). La stylistique des genres, Langue française, 135(135), 33-49.

Eco, U. (1985). Lector in fabula ou la coopération interprétative dans les textes narratifs. Paris : B. Grasset.

Jacquinot, G., \& Le Blanc, G. (1996). Les genres télévisuels dans l'enseignement. Paris : CNDP, Hachette éducation.

Jost, F. (1997). La promesse des genres. Réseaux, 81(15), 11-31.

Kerbrat-Orecchioni, C. (1980). L'énonciation, De la subjectivité dans le langage, Paris: Armand Colin.

Mondoloni, E. (2011). Les téléspectateurs préscolaires : des publics. Communication, lettres et sciences du langage, 5, 30-44.

Nel, N. (1997). Généricité, séquentialité, esthétique télévisuelles. Réseaux, 81(15), 3346.

Soulages, J.-C. (2007). Les rhétoriques télévisuelles : le formatage du regard. Bruxelles : De Boeck, Paris : INA. 